\section{Estimadores de mezcla amerindia basados en métodos serológicos y moleculares}

\author{
IVÁN PULGAR-ALARCÓN ${ }^{1, a}$, MACARENA FUENTES-GUAJARDO \\ ELENA LLOP ${ }^{3, a}$, FRANCISCO ROTHHAMMER ${ }^{2, b}$
}

\section{Amerindian admixture estimation based on serological and molecular methods}

Background: Amerindian admixture is an important parameter to consider in epidemiological studies in American countries, to make a proper selection of cases and controls. Aim: To compare Amerindian admixture estimates obtained using $A B O^{\star} A$ and $A B O^{\star} O$ blood group alleles and ancestral identity markers (AIMs) in the mixed Chilean population. Subjects and Methods: Amerindian admixture rates were determined in 720 Chilean volunteers residing in Arica and born in the 15 regions of the country, using $A B O^{\star} O$ and $A B O^{\star} A$ alleles and 40 AIMs selected from more than 500,000 single nucleotide polymorphisms (SNP's). Results: Mean admixture estimates obtained using $A B O^{*} O$ and $A B O^{*} A$ alleles and AIM's were $35,47 \%$ and $48 \%$ respectively. There was concordance in estimates, with the exception of the admixture based on $A B O^{*} O$ allele and AIMs. Conclusions: In Chile, Amerindian admixture estimates obtained using $A B O^{*} A$ could be used as an alternative to AIMs in justified cases provided the sample size is reasonably large.

(Rev Med Chile 2015; 143: 439-443)

Key words: American Native; Continental Ancestry Group; Chile, ABO Blood-Group System; Indians, South American.
${ }^{1}$ Centro de Investigaciones de Hombre en el Desierto, Arica,

Chile.

2Instituto de Alta Investigación Universidad de Tarapacá, Arica, Chile.

${ }^{3}$ Programa de Genética Humana ICBM Facultad de Medicina Universidad de Chile.

aLicenciado en Tecnología Médica, mención Laboratorio Clínico, Hematología y Banco de Sangre.

${ }^{b}$ Doctor en Ciencias.

Recibido el 8 de agosto de 2014, aceptado el 6 de marzo de 2015 .

Correspondencia a:

Francisco Rothhammer Instituto de Alta Investigación Universidad de Tarapacá. Calle Antofagasta 1520, Arica, Chile.

Teléfono: 58-2205073

Fax: 58-2255371

frothham@med.uchile.cl
$\mathrm{D}$ urante los casi 300 años en que Chile fue colonia militar española, ingresó al país un numeroso contingente de soldados de origen europeo que determinó un temprano enlace asimétrico de varones europeos y mujeres amerindias, que junto con la incorporación posterior de un grupo importante de esclavos africanos, originó la población mestiza que rápidamente llegaría a ser mayoría ${ }^{1,2}$.

La determinación de índices de mezcla utilizando los grupos sanguíneos clásicos ha sido ampliamente empleada en epidemiología ${ }^{3}$. Como es sabido, el sistema ABO, descubierto por Karl Landsteiner en 1900, está compuesto por los antígenos A y B, y los correspondientes anticuerpos contra estos antígenos ${ }^{4}$. El gen $\mathrm{ABO}$, ubicado en el cromosoma 9, posee tres alelos A, B y O, que varían de acuerdo a las sustituciones de nucleótidos, las cuales determinan las especificidades de las enzimas para las cuales codifican. Durante los últimos veinte años, la aplicación de técnicas de biología molecular ha sido incorporada gradualmente a la investigación biomédica, incluyendo recientemente la estimación supuestamente más precisa de índices de mezcla a partir de marcadores de identidad ancestral (MIA's) (AIM's en inglés) ${ }^{5}$ que son seleccionados a partir de un conjunto de polimorfismos de secuencia única (PSU's) (SNP's en inglés). No obstante, el elevado costo en equipos e insumos que significa la implementación de este método, determina que la técnica serológica tradicional siga utilizándose. El objetivo 
de este estudio es comparar los índices de mezcla estimados mediante los alelos del sistema $\mathrm{ABO}$ $\left(\mathrm{ABO}^{\star} \mathrm{O}\right.$ y $\left.\mathrm{ABO}^{\star} \mathrm{A}\right)$ y los MIA's, para determinar la confiabilidad del tradicional método serológico con respecto a la utilización de las nuevas técnicas moleculares.

\section{Sujetos y Métodos}

El estudio incluyó la participación de 720 voluntarias y voluntarios chilenos nacidos en las 15 regiones de Chile, mayores de 18 años, con residencia actual en la ciudad de Arica. Fueron incorporados al estudio aquellos participantes que decidieron participar después de recibir información detallada sobre los objetivos de éste mediante charlas públicas y folletos. Los voluntarios firmaron un consentimiento informado aprobado por el Comité de Ética/Bioética de la Universidad de Tarapacá y completaron una encuesta con la finalidad de obtener información sobre su ancestría. Mediante punción venosa se obtuvieron $10 \mathrm{ml}$ de sangre que se utilizó para realizar la fenotipificación del sistema $\mathrm{ABO}$ siguiendo las recomendaciones para la clasificación sanguínea del Instituto de Salud Pública de Chile ${ }^{6}$. Para estimar la frecuencia génica, se utilizó el programa S2 ABOESTIMA$\mathrm{TOR}^{7}$. El índice de mezcla amerindia se calculó de acuerdo al método de Bernstein a partir de las frecuencias de un único alelo para poblaciones dihíbridas descrito por Chakaborty $(1985)^{8,9}$. Debido a que el alelo B presenta frecuencias extremadamente bajas, se realizó el cálculo solamente para los alelos A y O. ADN genómico se obtuvo mediante el protocolo de extracción de Lahiri y Nurnberger ${ }^{10}$. Los MIA's fueron seleccionados entre los polimorfismos nucleotídicos únicos (PNU) por presentar frecuencias marcadamente diferentes entre las poblaciones involucradas en la estimación del índice de mezcla. Los PNU's se encuentran repartidos en el genoma en zonas que pueden o no codificar proteínas y ocurren, por definición, con una frecuencia relativa de al menos $1 \%$. Los estimadores de índices de mezcla obtenidos con técnicas moleculares tienen un nivel mayor de precisión debido a que en principio deberían cumplir en mejor forma los supuestos que es preciso realizar para utilizar el método de Bernstein, es decir, que los marcadores utilizados en el cálculo no hayan cambiado de frecuencia en las poblaciones ancestrales e híbridas debido a eventos mutacionales excepcionales, presiones selectivas y/o deriva genética. La estimación de ancestría de los voluntarios se realizó a partir de 40 MIA's seleccionados de entre cerca de 500.000 $\mathrm{PNU}^{\prime} \mathrm{s}^{11,12}$. El listado completo de MIA's utilizados y una discusión sobre la justificación de su elección se encuentra en Fuentes y cols. ${ }^{13}$. La genotipificación fue realizada por KBiosciences ${ }^{14}$ y el porcentaje individual de los componentes americano y europeo fue estimado con el programa computacional ADMIXTURE usando datos de genotipos publicados para las poblaciones parentales ${ }^{11-12}$. El nivel de significación de las comparaciones entre la tipificación de genes $\mathrm{ABO}$ y de MIA's, se determinó mediante la prueba de $t$ de Student para muestras relacionadas utilizando el programa IBM SPSS Statistics $19^{15-16}$. Además adelantándonos a que la condición de normalidad no se cumpla para la distribución de los índices de mezcla, utilizamos la prueba no paramétrica de Wilcoxon obteniendo sin embargo, resultados semejantes.

\section{Resultados}

La distribución fenotípica de los grupos $\mathrm{ABO}$ en las 15 Regiones de Chile indica, que O presenta la frecuencia más alta en todas las regiones (Tabla 1). Los promedios regionales de frecuencias alélicas sirvieron de base para obtener los promedios nacionales ponderados, siendo éstos para $\mathrm{ABO}^{\star} \mathrm{A}$ $0,1396 \pm 0,0095$, para $\mathrm{ABO}^{\star} \mathrm{B} 0,0766 \pm 0,0071$ y para $\mathrm{ABO}^{\star} \mathrm{O} 0,7838 \pm 0,0112$. La comparación de los índices de mezcla basados en las frecuencias de $\mathrm{ABO}^{\star} 0, \mathrm{ABO}^{\star} \mathrm{A}$ y $\mathrm{PNU}$ 's revela que el aporte americano promedio para Chile varía cuando es calculado utilizando $\mathrm{ABO}^{\star} \mathrm{O}(0,349 \pm 0,154)$, $\mathrm{ABO}^{\star} \mathrm{A}(0,469 \pm 0,153)$ o los MIA's $(0,482 \pm$ $0,50)$. Resulta evidente que se asemejan más los resultados obtenidos con el alelo $\mathrm{ABO}^{\star} \mathrm{A}$ y los MIA's. La utilización de la prueba de t de Student para promedios dependientes, como la prueba no paramétrica de Wilcoxon ratifican esta tendencia al ser esta comparación la única no significativa. Para algunas regiones como Atacama, Coquimbo, Los Ríos, Aysén y Magallanes el tamaño de la muestra es reducido, factor que debe ser considerado al observar los resultados separadamente por regiones (Tabla 2). 
Tabla 1. Frecuencias génicas regionales y nacionales de los grupos sanguíneos ABO en Chile

\begin{tabular}{|lrccc|}
\hline Región & $\mathbf{n}$ & A & Frecuencia génica & B \\
\hline Arica Parinacota & 142 & $0,1163 \pm 0,02$ & $0,0849 \pm 0,02$ & $0,7988 \pm 0,02$ \\
\hline Tarapacá & 38 & $0,0827 \pm 0,03$ & $0,0827 \pm 0,03$ & $0,8346 \pm 0,04$ \\
\hline Antofagasta & 53 & $0,1973 \pm 0,04$ & $0,0779 \pm 0,03$ & $0,7247 \pm 0,05$ \\
\hline Atacama & 12 & $0,1869 \pm 0,08$ & $0,1365 \pm 0,07$ & $0,6763 \pm 0,10$ \\
\hline Coquimbo & 15 & $0,1058 \pm 0,06$ & $0,0340 \pm 0,03$ & $0,8602 \pm 0,06$ \\
\hline Valparaíso & 28 & $0,156 \pm 0,05$ & $0,0747 \pm 0,04$ & $0,7692 \pm 0,06$ \\
\hline Región Metropolitana & 155 & $0,1275 \pm 0,02$ & $0,0984 \pm 0,02$ & $0,7742 \pm 0,02$ \\
\hline O'Higgins & 27 & $0,1599 \pm 0,05$ & $0,0375 \pm 0,03$ & $0,8026 \pm 0,06$ \\
\hline Maule & 48 & $0,1460 \pm 0,04$ & $0,0645 \pm 0,03$ & $0,7895 \pm 0,04$ \\
\hline BíoBío & 105 & $0,1543 \pm 0,03$ & $0,0587 \pm 0,02$ & $0,7870 \pm 0,03$ \\
\hline Araucanía & 52 & $0,1112 \pm 0,03$ & $0,0590 \pm 0,02$ & $0,8297 \pm 0,04$ \\
\hline Los Ríos & 14 & $0,2002 \pm 0,08$ & $0,0749 \pm 0,05$ & $0,7247 \pm 0,09$ \\
\hline Los Lagos & 20 & $0,3338 \pm 0,08$ & $0,0520 \pm 0,04$ & $0,6140 \pm 0,09$ \\
\hline Aysén & 5 & $0,1063 \pm 0,10$ & $0,1063 \pm 0,10$ & $0,7874 \pm 0,13$ \\
\hline Magallanes & 6 & $0,1855 \pm 0,12$ & $0,0881 \pm 0,08$ & $0,7262 \pm 0,14$ \\
\hline Total Chile & 720 & $0,1396 \pm 0,09$ & $0,0766 \pm 0,01$ & $0,7838 \pm 0,01$ \\
\hline
\end{tabular}

Tabla 2. Comparación del índice de mezcla por región, determinado por la fenotipificación ABO y el análisis de AIM's

\begin{tabular}{|lcccc|}
\hline Región & $\mathbf{n}$ & $\mathbf{A B O} \boldsymbol{*}^{\mathbf{O}}$ & ABO*A & SNP's \\
\hline Arica Parinacota & 142 & 0,011 & 0,593 & 0,511 \\
\hline Tarapacá & 38 & 0,530 & 0,711 & 0,531 \\
\hline Antofagasta & 53 & 0,218 & 0,310 & 0,508 \\
\hline Atacama & 12 & 0,080 & 0,347 & 0,511 \\
\hline Coquimbo & 15 & 0,603 & 0,630 & 0,421 \\
Valparaíso & 28 & 0,344 & 0,455 & 0,406 \\
\hline Región Metropolitana & 155 & 0,358 & 0,554 & 0,405 \\
\hline O' Higgins & 27 & 0,439 & 0,441 & 0,430 \\
\hline Maule & 48 & 0,402 & 0,490 & 0,453 \\
\hline BíoBío & 105 & 0,395 & 0,460 & 0,532 \\
\hline Araucanía & 52 & 0,516 & 0,611 & 0,537 \\
\hline Los Ríos & 14 & 0,218 & 0,300 & 0,502 \\
\hline Los Lagos & 20 & 0,097 & 0,167 & 0,555 \\
\hline Aysén & 5 & 0,396 & 0,628 & 0,411 \\
\hline Magallanes & 6 & 0,222 & 0,351 & $0,4822 \pm 0,05$ \\
\hline Total Chile & 720 & $0,3497 \pm 0,15$ & $0,4699 \pm 0,15$ & 0 \\
\hline
\end{tabular}




\section{Discusión}

A diferencia de otros estudios de mezcla americana evitamos utilizar muestras de bancos de sangre debido a que frecuentemente en estas instituciones se buscan donantes portadores de grupos poco frecuentes para prevenir urgencias futuras, procedimiento que viola el supuesto de aleatoriedad y en consecuencia puede sesgar los estimadores de frecuencias génicas y de resultados basados en éstas ${ }^{17,18}$. Los voluntarios participantes en este estudio fueron agrupados de acuerdo a su lugar de nacimiento con el objeto de obtener una estimación del grado de variación regional de los índices de mezcla. Al ser comparados los valores de mezcla americana para las regiones del Norte Árido, del Norte Semi-Árido, la Zona Centro-Sur y la Patagonia se obtienen resultados concordantes con el poblamiento inicial de Chile y los desplazamientos poblacionales posteriores, exhibiendo el Norte Árido los valores de mezcla más altos, seguido de la Patagonia, el Norte Semi-Árido y la Zona Centro-Sur. Los mayores porcentajes de mezcla se concentran en el extremo norte y sur del país, mientras que en las regiones centrales son más altos los porcentajes de mezcla europea. Resulta notorio constatar que la población mixta chilena ha sido objeto de menos estudios publi$\operatorname{cados}^{13,13,17,18,19}$ que las poblaciones originarias, probablemente debido al interés antropológico que han suscitado estas últimas ${ }^{5-19}$. Los promedios de frecuencias génicas nacionales obtenidos son semejantes a los obtenidos en poblaciones urbanas de algunas regiones del país con anterioridad, donde $O$ varía entre 0,769 y 0,84 , A entre 0,102 y 0,72 y $B$ entre 0,046 y $0,080^{20}$. Algunos de estos resultados fueron obtenidos en muestras pequeñas, factor que es preciso considerar debido a que el tamaño de la muestra cuando es muy pequeña puede aumentar la varianza de las frecuencias génicas y sesgar la estimación de los índices de mezcla.

Resumiendo, los índices de mezcla obtenidos con el alelo $\mathrm{ABO}^{\star} \mathrm{A}$ son más semejantes a los obtenidos con los MIA's que aquellos calculados con base en el alelo $\mathrm{ABO}^{\star} \mathrm{O}$. Cuando el tamaño de la muestra es razonablemente grande, la obtención de índices de mezcla empleando el alelo $\mathrm{ABO}^{\star} \mathrm{A}$ no difiere estadísticamente de los resultados obtenidos con marcadores moleculares a diferencia del alelo $\mathrm{ABO}^{\star} 0$ (Tabla 2) pudiendo en casos justificados constituir una alternativa al uso de MIA's.

\section{Referencias}

1. Valenzuela C, Acuña M, Harb Z. Gradiente Socioeconómico en la Población Chilena. Rev Med Chile 1987; 115: 295-9.

2. Gallego J. La Esclavitud en la América española, Madrid: Ed. Encuentros, 2005, p. 19.

3. Aguirre R, Armanet L, Castillo S, Cifuentes L, CruzCoke R, Etcheverry A, et al. Identidad genética y prueba de paternidad, Santiago, Chile: Editorial Universitaria. 1996. Cap. 5 p. 89-120.

4. Daniels G, Fletcher A, Garratty G, Henry S, Jørgensen J, Judd W, et al. Blood group terminology 2004: from the International Society of Blood Transfusion committee on terminology for red cell surface antigens. Vox Sang 2004; 87: 304-16.

5. De Saint Pierre M, Gandini F, Perego H, Bodner M, Gómez-Carballa A, Corach D, et al. Arrival of Paleo-Indians to the Southern Cone of South America: New Clues from Mitogenomes. Plos One 2012: 51311.

6. Aburto A. Recomendaciones para la clasificación sanguínea ABO. Instituto de Salud Pública de Chile. 2012. http://www.ispch.cl/sites/default/files/documento/2013/03/clasificacion\%20sanguinea \%20-\%20 30012013A.pdf [Consultado el 4 de junio de 2014].

7. Silva P. Facultad de Ciencias Universidad de Lisboa. Disponible en: http://webpages.fc.ul.pt/ pjns/Soft/ ABOestimator/ [Consultado el 4 de junio de 2014].

8. Bernstein F. En: Die geographische Verteilung der Blutgruppen und ihreanthropologische Bedeutung. In Comitato Italiano per lo Studio dei problemi della populazione. Rome: Instituto Poligráfico dello Stato 1931; 227-43.

9. Chakraborty R. Gene identity in racial hybrids and estimation of admixture rates. En: Ahuja YR, Neel JV (Eds) Genetic differentiation in human and other animal populations. Indian Anthropological Association, Delhi 1985 p. 171-80.

10. Lahiri D, Nurnberger J. A rapid non-enzimatic method for preparation of HMW DNA from blood for RFLP studies. Nucl Acids Res 1991; 19: 5444.

11. Alexander D, Novembre J, Lange K. Fast model-based estimation of ancestry in unrelated individuals. Genome Res 2009; 19: 1655-64.

12. Reich D, Patterson N, Campbell D, Tandon A, Mazieres S, Ray N, et al. Reconstructing Native American population history. Nature 2012; 488: 370-4.

13. Fuentes M, Pulgar I, Gallo C, Bortolini MC, Canizales-Quinteros S, Bedoya G, et al. Geografía génica de Chile. Distribución regional de los aportes genéticos americanos, europeos y africanos. Rev Med Chile 2014; 142: 281-9. 
14. Hoddesdon, Herts, United Kingdom; http://www.kbioscience.co.uk/.

15. Dawson B, Trapp R. Basic and Clinical Biostatistics, Lange Medical Books McGraw-Hill 2004. 4th edition.

16. Pardo A, Ruiz M. Análisis de Datos: En Ciencias Sociales y de la Salud II, Editorial Síntesis, Madrid 2010. p 174-8.

17. Cifuentes L, Valenzuela C, Cruz-Coke R, Armanet L, Lyng C, Harb Z. Caracterización genética de la población hospitalaria de Santiago. Rev Med Chile 1988; 116 : 28-33.

18. Pinto G, Ilic P, Paredes L, González T, Marinovic F,
Valenzuela CY. Frecuencias Fenotípicas y Génicas de los Sistemas Sanguíneos ABO y Rhesus en Clínica Alemana (Santiago, Chile). Rev Med Chile 1980; 109: 1209-11.

19. Acuña M, Llop E, Rothhammer F. Composición genética de la población chilena. las comunidades rurales de los valles de Elqui, Limarí y Choapa. Rev Med Chile 2000; 128 (6): 593-600.

20. Llop E. Genetic composition of Chilean aboriginal populations: HLA and other genetic marker variation. Am J Phys Anthropol 1996; 101: 325-32. 